University of Nebraska - Lincoln

DigitalCommons@University of Nebraska - Lincoln

June 1974

\title{
Photoelectron angular distributions, cross sections, and branching ratios for atomic oxygen
}

Anthony F. Starace

University of Nebraska-Lincoln, astarace1@unl.edu

Steven T. Manson

Georgia State University, Atlanta, Georgia

David J. Kennedy

Georgia Institute of Technology, Atlanta, Georgia

Follow this and additional works at: https://digitalcommons.unl.edu/physicsstarace

Part of the Physics Commons

Starace, Anthony F.; Manson, Steven T.; and Kennedy, David J., "Photoelectron angular distributions, cross sections, and branching ratios for atomic oxygen" (1974). Anthony F. Starace Publications. 4. https://digitalcommons.unl.edu/physicsstarace/4

This Article is brought to you for free and open access by the Research Papers in Physics and Astronomy at DigitalCommons@University of Nebraska - Lincoln. It has been accepted for inclusion in Anthony F. Starace Publications by an authorized administrator of DigitalCommons@University of Nebraska - Lincoln. 


\title{
Photoelectron angular distributions, cross sections, and branching ratios for atomic oxygen
}

\author{
Anthony F. Starace* \\ Behlen Laboratory of Physics, The University of Nebraska, Lincoln, Nebraska 68508 \\ Steven T. Manson ${ }^{\dagger}$ \\ Department of Physics, Georgia State University, Atlanta, Georgia 30303 \\ David J. Kennedy ${ }^{\ddagger}$ \\ School of Physics, Georgia Institute of Technology, Atlanta, Georgia 30332
}

(Received 30 January 1974)

\begin{abstract}
Theoretical calculations of the angular distribution of photoelectrons ejected from the $2 p$ subshell of atomic oxygen for photoelectron kinetic energies $0 \leq \epsilon \leq 30$ Ry are presented using both Hartree-Fock (HF) and Herman-Skillman (HS) wave functions. Owing to the weakness of anisotropic electron-ion interactions, as evidenced by small differences between HF photoelectron phase shifts for alternative outgoing channels, the simple HS calculation of the angular-distribution asymmetry parameter is found to agree to within $\varsigma 0.1$ with the HF calculations. Photoionization cross sections and photoelectron branching ratios have also beem computed in both the HF and HS approximation for the $2 p$ subshell of atomic oxygen in the wavelength range $910 \leq \lambda \leq 100 \AA$. Comparison is made with other theoretical calculations and with the experimental branching-ratio measurements of Samson and Petrosky at $\lambda=584.3 \AA$.
\end{abstract}

\section{INTRODUCTION}

Dill, Manson, and Starace ${ }^{1}$ have shown that the Cooper-Zare theory ${ }^{2}$ for photoelectron angular distributions, while correct for closed-shell atoms, is not appropriate for open-shell atoms except in those cases where the phase shifts for alternative photoelectron channels are the same. We present here theoretical calculations that confirm atomic oxygen as one of these special cases. We find that the use of Herman-Skillman ${ }^{3}$ (HS) wave functions and the Cooper-Zare theory provides angular distribution asymmetry parameters that are in substantial agreement with those obtained using Hartree-Fock (HF) wave functions and a more rigorous theory ${ }^{1}$ for photoelectron angular distributions. We expect similar results for the other atoms having open $2 p$ subshells. Thus, the angular distributions of photoelectrons from $2 p$ subshells calculated by Manson ${ }^{4}$ using HS wave functions and the Cooper-Zare theory should prove reliable.

We present also new calculations, using both HS and HF wave functions, of the total and partial photoionization cross sections of atomic oxygen. Because these cross sections are of fundamental importance for upper atmosphere physics, they have been calculated by many authors..$^{5-10}$ Following $\mathrm{Henry}^{7}$ we plot also photoelectron branching ratios since these are the quantities measured directly in photoelectron spectroscopy. At $\lambda=584.3$ $\AA$ our branching ratios are in reasonable agree ment with both the experimental values of Samson and Petrosky ${ }^{11}$ and the more accurate close-coupling calculations of Henry. ${ }^{7}$ The calculated angular distributions, cross sections, and branching ratios presented in this paper thus extend and complement existing theoretical data on the photoionization of atomic oxygen.

\section{THEORY}

Dill and Fano ${ }^{12-14}$ have expressed the differential cross section for photoionization of an unpolarized target as an incoherent sum over contributions corresponding to alternative values of the angular momentum $\vec{j}_{t}$ transferred by the incident photon to the target:

$$
\frac{d \sigma}{d \Omega}=\sum_{j_{t}} \frac{\sigma\left(j_{t}\right)}{4 \pi}\left[1+\beta\left(j_{t}\right) P_{2}(\cos \theta)\right] .
$$

Detailed expressions for the partial cross sections $\sigma\left(j_{t}\right)$ and asymmetry parameters $\beta\left(j_{t}\right)$ are given in Ref. 14 in terms of scattering amplitudes $S_{l}\left(j_{t}\right)$, where $l$ is the orbital angular momentum of the outgoing photoelectron. The experimentally measured asymmetry parameter $\beta$ is then given by the following weighted average $e^{14}$ :

$$
\beta=\left(\sum_{j_{t}} \sigma\left(j_{t}\right) \beta\left(j_{t}\right)\right) / \sum_{j_{t}} \sigma\left(j_{t}\right) .
$$

For the particular case of $L S$ coupling the general photoionization process via electric dipole interaction,

$A\left(l_{0}^{n} L_{0} S_{0} J_{0}\right)+h \nu \rightarrow A^{+}\left(l_{0}^{n-1} L_{c} S_{c} J_{c}\right)+e^{-}(l, s, j)$, 
has been shown by Dill, Manson, and Starace ${ }^{1}$ to have the following amplitude for transfer of $j_{t}$ units of angular momentum (when the separation between fine-structure levels $J_{c}$ is ignored):

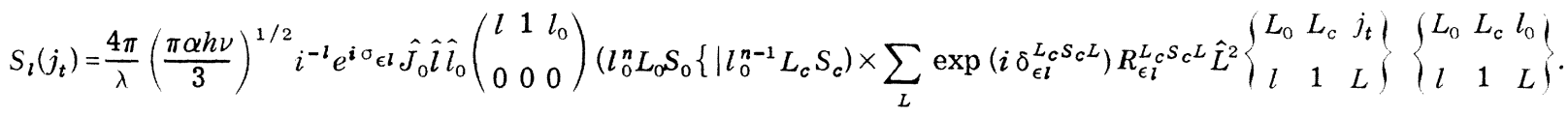

Here $\sigma_{\epsilon l}$ is the Coulomb phase shift, dependent on the photoelectron orbital momentum $l$ and kinetic energy $\epsilon, \hat{x} \equiv(2 x+1)^{1 / 2}, \nu \lambda=c$, and $\delta_{\epsilon l}^{L} c^{c} c^{L}$ is the photoelectron phase shift relative to Coulomb waves. The radial dipole matrix element is given by

$$
R_{\epsilon l}^{L_{c} S_{c}{ }^{L}} \equiv \int_{0}^{\infty} \psi_{i}\left(n_{0} l_{0} L_{0} S_{0} \mid r\right) r \psi_{f}\left(\epsilon l L_{c} S_{c} L \mid r\right) d r
$$

and the final-state continuum wave function $\psi_{f}$ is normalized per unit energy.

Three points emphasized in Ref. 1 are important for understanding the calculations presented below. First, only in the limit that the final state wave functions $\psi_{f}$ (and thus the phase shifts $\delta_{\epsilon l}^{L_{c} S_{c} L}$ and dipole matrix elements $R_{\epsilon l}^{L_{c} s_{c} L}$ ) are independent of $L$, and hence of $L_{c} S_{c}$, does the asymmetry parameter $\beta$ in Eq. (2) reduce to the form obtained by Cooper and Zare. ${ }^{2}$ For in this special case the sum over $L$ in Eq. (4) may be performed analytically, yielding a $\delta$ function that restricts $j_{t}$ to the single value $j_{t}=l_{0}$. In other words, the Cooper-Zare formula obtains only in the limit when there is no dynamical coupling of the orbital motion of the photoelectron to the net orbital motion of the residual ion. Second, Ref. 14 shows that the asymmetry parameter $\beta$ depends on products of the scattering amplitudes in Eq. (4): specifically, on $\left|S_{+}\left(j_{t}\right)\right|^{2},\left|S_{-}\left(j_{t}\right)\right|^{2}$, and $\left[S_{+}\left(j_{t}\right) S_{-}^{\dagger}\left(j_{t}\right)+\right.$ c.c. $]$, where the subscripts \pm indicate $l=j_{t} \pm 1$. Each of these products contains cross terms having a factor

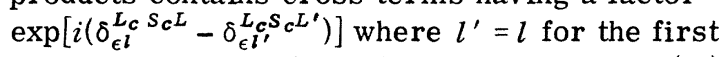
two products and $l^{\prime}=l-2$ for the product $S_{+}\left(j_{t}\right)$ $\times S_{-}^{\dagger}\left(j_{t}\right)$. The Cooper-Zare theory, however, assumes the dynamical phase shifts to be independent of $L_{c}, S_{c}$, and $L$; hence only the product $S_{+}\left(j_{t}\right)$ $\times S_{-}^{\dagger}\left(j_{t}\right)$ involves a phase shift difference, which occurs in a factor $\exp \left[i\left(\delta_{\epsilon l}-\delta_{\epsilon l}\right)\right]$ for $l^{\prime}=l-2$. Therefore examination of the phase-shift differences for alternative photoelectron channels $L_{c} S_{c} L$ provides an estimate of the magnitude of anisotropic electron-ion interactions and hence of the validity of the Cooper-Zare formula. Third, for open-shell atoms the Cooper-Zare theory predicts the asymmetry parameters for alternative ion levels $L_{c} S_{c}$ to be identical when plotted as a function of photoelectron kinetic energy $\epsilon$. Again, the validity of this result depends on the strength of anisotropic electron-ion interactions.

We are concerned here with the following photoionization processes in atomic oxygen:

$$
\mathrm{O}\left(2 p^{4}{ }^{3} P\right)+h \nu \rightarrow \mathrm{O}^{+}\left(2 p^{34} S,{ }^{2} D,{ }^{2} P\right)+e^{-} .
$$

Binding energies for the ${ }^{4} S,{ }^{2} D$, and ${ }^{2} P$ ion terms are respectively 0.50079 a.u. $(909.83 \AA), 0.62300$ a.u. $(731.35 \AA)$, and 0.68519 a.u. $(664.97 \AA) .{ }^{15}$ These experimental energies were used in computing the theoretical cross sections presented below.

Our discrete HF single-particle orbitals for the neutral atom and for the ion were obtained from the tabulation of Clementi. ${ }^{16}$ Continuum HF orbitals were obtained by the procedures described fully in Refs. 6 and 17. These continuum wave functions depend on both the ionic term level and on the total orbital angular momentum and thus the asymmetry parameter in Eq. (2) must be computed us ing the scattering amplitude in Eq. (4).

The HS continuum wave functions, on the other hand, do not depend on the ionic term levels or on the total orbital angular momentum and thus the Cooper-Zare formula for the asymmetry parameter is appropriate. These wave functions are

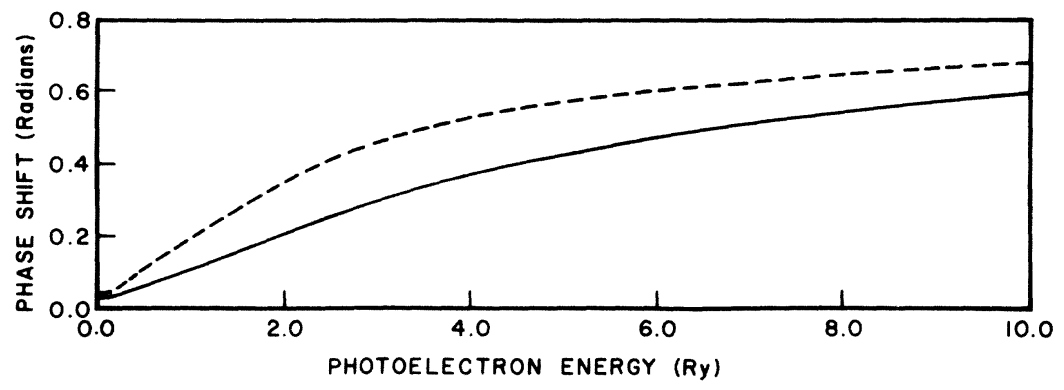

FIG. 1. Hartree-Fock $d$ wave phase shifts $\delta_{\varepsilon d}^{L_{c} S_{c} L}$ for the ${ }^{2} D$ ion term vs photoelectron kinetic energy $\epsilon$ for two allowed values of $L$. Solid line corresponds to $L=0$ [i.e., the state $\left.2 p^{3}\left({ }^{2} D\right) \in d^{3} S\right]$ and the dashed line to $L=2$ [i.e., the state $2 p^{3}\left({ }^{2} D\right) \in d^{3} D$ ] 
TABLE I. HF asymmetry parameters for the reactions $\mathrm{O}\left(2 p^{4}{ }^{3} P\right)+h \nu \rightarrow \mathrm{O}^{+}\left(2 p^{3}{ }^{4} S,{ }^{2} D,{ }^{2} P\right)$ $+e^{-}$as a function of photoelectron kinetic energy $\epsilon$ using dipole length (velocity) formula and comparison with HS asymmetry parameter.

\begin{tabular}{rcccc}
\hline \hline$\epsilon(\mathrm{Ry})$ & $\beta\left({ }^{3} P \rightarrow{ }^{4} S\right)$ & $\beta\left({ }^{3} P \rightarrow{ }^{2} D\right)$ & $\beta\left({ }^{3} P \rightarrow{ }^{2} P\right)$ & HS $\beta$ \\
\hline 0.00 & $-0.243(-0.140)$ & $-0.205(-0.114)$ & $-0.199(-0.89)$ & -0.110 \\
0.05 & $-0.247(-0.138)$ & $-0.248(-0.149)$ & $-0.233(-0.114)$ & -0.105 \\
0.10 & $-0.091(0.010)$ & $-0.104(-0.011)$ & $-0.086(0.025)$ & +0.033 \\
0.20 & $0.183(0.267)$ & $0.159(0.237)$ & $0.181(0.274)$ & 0.277 \\
0.40 & $0.548(0.603)$ & $0.520(0.572)$ & $0.544(0.606)$ & 0.605 \\
0.60 & $0.784(0.815)$ & $0.756(0.787)$ & $0.782(0.817)$ & 0.799 \\
0.80 & $0.944(0.958)$ & $0.918(0.932)$ & $0.946(0.961)$ & 0.946 \\
1.00 & $1.061(1.062)$ & $1.038(1.038)$ & $1.067(1.065)$ & 1.052 \\
1.50 & $1.250(1.230)$ & $1.230(1.211)$ & $1.261(1.234)$ & 1.218 \\
2.00 & $1.360(1.329)$ & $1.342(1.313)$ & $1.376(1.335)$ & 1.317 \\
3.00 & $1.473(1.437)$ & $1.458(1.427)$ & $1.495(1.444)$ & 1.424 \\
4.00 & $1.523(1.489)$ & $1.508(1.482)$ & $1.545(1.496)$ & 1.476 \\
6.00 & $1.541(1.521)$ & $1.525(1.518)$ & $1.560(1.525)$ & 1.505 \\
8.00 & $1.519(1.509)$ & $1.502(1.507)$ & $1.532(1.509)$ & 1.495 \\
10.00 & $1.479(1.479)$ & $1.462(1.477)$ & $1.485(1.475)$ & 1.461 \\
20.00 & $1.264(1.267)$ & $1.258(1.265)$ & $1.259(1.258)$ & 1.250 \\
30.00 & $1.069(1.069)$ & $1.067(1.068)$ & $1.059(1.060)$ & 1.075 \\
\hline \hline
\end{tabular}

computed in the tabulated potential field of Herman and Skillman, ${ }^{3}$ which does not account for core relaxation. Discrete states for both the ion and the atom were taken to be the tabulated HS neutralatom discrete orbitals. In computing the partial cross sections for photoionization to alternative ionic term levels the only difference is the experimental binding energy used: For a given photoelectron kinetic energy the wave functions employed are identical. Further details on the HS independent particle model are provided by Manson and Cooper. ${ }^{18}$

TABLE II. HF asymmetry parameters for the reactions $\mathrm{O}\left(2 p^{41} D\right)+h \nu \rightarrow \mathrm{O}^{+}\left(2 p^{32} D,{ }^{2} P\right)+e^{-}$and $\mathrm{O}\left(2 p^{4}{ }^{1} S\right)$ $+h \nu \rightarrow \mathrm{O}^{+}\left(2 p^{32} P\right)+e^{-}$as a function of photoelectron kinetic energy $\epsilon$ using dipole length (velocity) formula.

\begin{tabular}{rccc}
\hline$\epsilon(\mathrm{Ry})$ & $\beta\left({ }^{1} D \rightarrow{ }^{2} D\right)$ & $\beta\left({ }^{1} D \rightarrow{ }^{2} P\right)$ & $\beta\left({ }^{1} S \rightarrow{ }^{2} P\right)$ \\
\hline 0.00 & $-0.202(-0.127)$ & $-0.155(-0.090)$ & $-0.184(-0.099)$ \\
0.05 & $-0.230(-0.147)$ & $-0.221(-0.147)$ & $-0.218(-0.125)$ \\
0.10 & $-0.088(-0.007)$ & $-0.092(-0.018)$ & $-0.081(0.009)$ \\
0.20 & $0.169(0.240)$ & $0.154(0.220)$ & $0.169(0.247)$ \\
0.40 & $0.519(0.569)$ & $0.498(0.547)$ & $0.509(0.564)$ \\
0.60 & $0.749(0.779)$ & $0.728(0.758)$ & $0.734(0.768)$ \\
0.80 & $0.907(0.921)$ & $0.888(0.903)$ & $0.888(0.906)$ \\
1.00 & $1.023(1.025)$ & $1.005(1.010)$ & $1.002(1.009)$ \\
1.50 & $1.209(1.193)$ & $1.197(1.184)$ & $1.188(1.177)$ \\
2.00 & $1.318(1.294)$ & $1.310(1.289)$ & $1.297(1.279)$ \\
3.00 & $1.430(1.406)$ & $1.427(1.408)$ & $1.412(1.396)$ \\
4.00 & $1.478(1.463)$ & $1.479(1.468)$ & $1.464(1.457)$ \\
6.00 & $1.498(1.504)$ & $1.502(1.511)$ & $1.489(1.503)$ \\
8.00 & $1.479(1.500)$ & $1.481(1.507)$ & $1.472(1.501)$ \\
10.00 & $1.448(1.475)$ & $1.450(1.481)$ & $1.443(1.478)$ \\
20.00 & $1.259(1.275)$ & $1.265(1.278)$ & $1.263(1.280)$ \\
30.00 & $1.077(1.078)$ & $1.080(1.081)$ & $1.085(1.084)$ \\
\hline \hline
\end{tabular}

Finally, the theory of atomic photoionization and appropriate formulas have been presented by Bates, ${ }^{19}$ Dalgarno, Henry, and Stewart, ${ }^{6}$ and Manson and Cooper. ${ }^{18}$

\section{RESULTS}

In Fig. 1 we have plotted HF phase shifts $\delta_{\epsilon d}^{L}{ }_{\epsilon d} S_{c} L$ as a function of photoelectron kinetic energy $\epsilon$ for the ${ }^{2} D$ ion term and for two values of $L$. These two phases differ from one another by about 0.16 $\mathrm{rad}$ in the energy region $2.0 \leqslant \epsilon \leqslant 4.0$. This difference is the largest found among any two oxygen phase shifts. It compares with phase-shift differences of up to $0.6 \mathrm{rad}$ found between different photoelectron channels in atomic sulfur. ${ }^{1}$ Because of these relatively small phase-shift differences in atomic oxygen, we expect the asymmetry parameter calculated with HS wave functions and the Cooper-Zare formula ${ }^{2}$ to be in reasonably good agreement with asymmetry parameters calculated with $\mathrm{HF}$ wave functions and the more rigorous angular distribution formulas of Ref. 1.

In Table I we compare our HF asymmetry parameters with the HS one as a function of photoelectron kinetic energy. Note that if the oxygen photoelectron phase shifts were all identical then the HF asymmetry parameters for each ionic term would be the same. As it is, we see that the differences between the HF asymmetry parameters for different ionic term levels are generally smaller than the length and velocity values for a given term level. If the $\mathrm{HF}$ length values are regarded as the correct ones, ${ }^{20}$ then the $\mathrm{HS}$ values are about 0.1 too high for $0.0 \leqslant \epsilon \leqslant 0.4 \mathrm{Ry}$, but are 


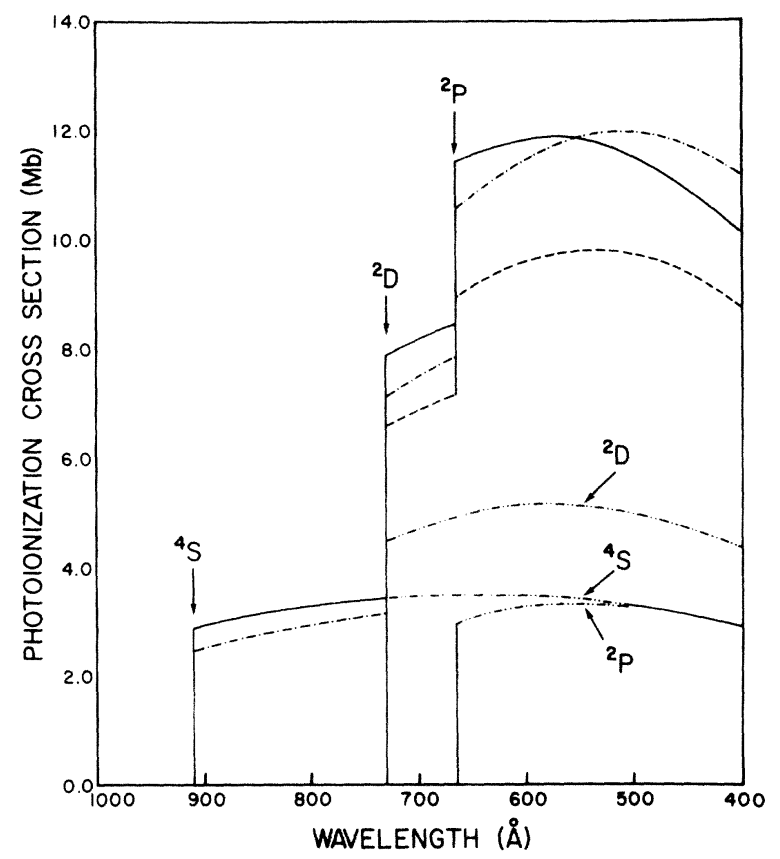

FIG. 2. Total and partial photoionization cross sections for atomic oxygen. Solid line: HS total cross section. Dash-dot line: HF (length) total cross section. Dashed line: HF (velocity) total cross section. Dash-double-dot line: HS partial cross sections. Note that for $910 \leq \lambda \leq 731$ the HS and $\mathrm{HF}$ velocity cross sections coincide.

in excellent agreement with HF for higher kinetic energies. In Table II we have listed HF asymmetry parameters for photoionization from the ${ }^{1} D$ and ${ }^{1} S$ excited term levels of atomic oxygen. These are also in agreement with the HS asymmetry parameter in Table $I$.

In Fig. 2 we have plotted HS and HF total cross sections for atomic oxygen. The HS partial cross sections are also shown and the HF partial cross sections are listed in Table III. We see that near each threshold the HS cross sections are higher than either HF length or HF velocity. For higher kinetic energies, the HS cross sections fall between HF length and velocity. Note that at the ${ }^{4} S$ threshold our HF length (velocity) cross section of $2.46(2.89)$ agrees best with the values $2.3(2.8)$ of Bates and Seaton ${ }^{5}$ rather than the values 2.7 (3.4) of Dalgarno, Henry, and Stewart. ${ }^{6}$ The differences among these three HF calculations probably arise from the different discrete orbitals used. Above the ${ }^{2} P$ threshold our HF length and velocity cross sections are slightly lower $(\simeq 0.2-0.3 \mathrm{Mb})$ than those of Ref. 6 but not as low as the more accurate close-coupling cross sections of Henry. ${ }^{7}$ It is unclear why the cross sections of Dalgarno, Henry, and Stewart ${ }^{6}$ and of this paper are in closer agreement with the absolute experimental measurements of Cairns and Samson ${ }^{21}$ than is the more complete calculation of Henry. ${ }^{7}$

Finally, in Fig. 3 we have plotted our HS photoelectron branching ratios $\sigma\left({ }^{2} D\right) / \sigma\left({ }^{4} S\right)$ and $\sigma\left({ }^{2} P\right) /$ $\sigma\left({ }^{4} S\right)$ and compared them with those of Henry. ${ }^{7}$ In Table III we also list the $\mathrm{HF}$ branching ratios we have obtained. For $731 \leqslant \lambda \leqslant 300 \AA$ our HF-length branching ratios agree nearly identically with those of HS. For $\lambda<300 \AA$ the HF-length branching ratios drop below the values obtained by Henry. ${ }^{7}$ The HF-velocity branching ratios are much lower than the HF-length ratios for $\lambda>300 \AA$ and generally equal to the length ratios below $300 \AA$. At $584.3 \AA$ both our HS- and HF-length branching ratios are equal. Our $\sigma\left({ }^{2} D\right) / \sigma\left({ }^{4} S\right)$ branching ratio of 1.48-1.49 compares with the experimental ratio of $1.57 \pm 0.14 .{ }^{11}$ Our $\sigma\left({ }^{2} P\right) / \sigma\left({ }^{4} S\right)$ ratio of $0.95-0.96$

TABLE III. HF partial cross sections and branching ratios for the reactions $\mathrm{O}\left(2 p^{4}{ }^{3} P\right)+h \nu$ $\rightarrow \mathrm{O}^{+}\left(2 p^{4} S,{ }^{2} D,{ }^{2} P\right)+e^{-}$as a function of wavelength using dipole length (velocity) formula.

\begin{tabular}{cccccc}
\hline \hline$\lambda(\AA)$ & $\sigma\left({ }^{4} S\right)$ & $\left.\sigma{ }^{2} D\right)$ & $\sigma\left({ }^{2} P\right)$ & $\sigma\left({ }^{2} D\right) / \sigma\left({ }^{4} S\right)$ & $\sigma\left({ }^{2} P\right) / \sigma\left({ }^{4} S\right)$ \\
\hline 909.8 & $2.463(2.892)$ & $\ldots$ & $\ldots$ & $\ldots$ & $\ldots$ \\
731.4 & $3.122(3.399)$ & $3.982(3.177)$ & $\ldots$ & $1.28(0.93)$ & $\ldots$ \\
665.0 & $3.285(3.450)$ & $4.578(3.685)$ & $2.682(1.806)$ & $1.39(1.07)$ & $0.82(0.52)$ \\
650.0 & $3.321(3.461)$ & $4.685(3.775)$ & $2.802(1.904)$ & $1.41(1.09)$ & $0.84(0.55)$ \\
600.0 & $3.374(3.400)$ & $4.948(3.985)$ & $3.122(2.173)$ & $1.47(1.17)$ & $0.93(0.64)$ \\
584.3 & $3.391(3.381)$ & $5.013(4.034)$ & $3.205(2.246)$ & $1.48(1.19)$ & $0.95(0.66)$ \\
550.0 & $3.400(3.308)$ & $5.142(4.129)$ & $3.315(2.348)$ & $1.51(1.25)$ & $0.97(0.71)$ \\
500.0 & $3.374(3.159)$ & $5.183(4.122)$ & $3.421(2.451)$ & $1.54(1.31)$ & $1.01(0.78)$ \\
450.0 & $3.275(2.943)$ & $5.085(3.992)$ & $3.400(2.449)$ & $1.55(1.36)$ & $1.04(0.83)$ \\
400.0 & $3.097(2.668)$ & $4.809(3.721)$ & $3.239(2.338)$ & $1.55(1.39)$ & $1.05(0.88)$ \\
350.0 & $2.827(2.332)$ & $4.336(3.307)$ & $2.926(2.116)$ & $1.53(1.42)$ & $1.04(0.91)$ \\
300.0 & $2.461(1.940)$ & $3.674(2.760)$ & $2.444(1.772)$ & $1.49(1.42)$ & $0.99(0.91)$ \\
250.0 & $1.979(1.496)$ & $2.813(2.092)$ & $1.829(1.335)$ & $1.42(1.40)$ & $0.92(0.89)$ \\
200.0 & $1.405(1.028)$ & $1.850(1.380)$ & $1.157(0.864)$ & $1.32(1.34)$ & $0.82(0.84)$ \\
150.0 & $0.815(0.588)$ & $0.991(0.751)$ & $0.591(0.457)$ & $1.21(1.28)$ & $0.72(0.78)$ \\
100.0 & $0.308(0.222)$ & $0.339(0.268)$ & $0.193(0.159)$ & $1.10(1.21)$ & $0.63(0.72)$ \\
\hline \hline
\end{tabular}




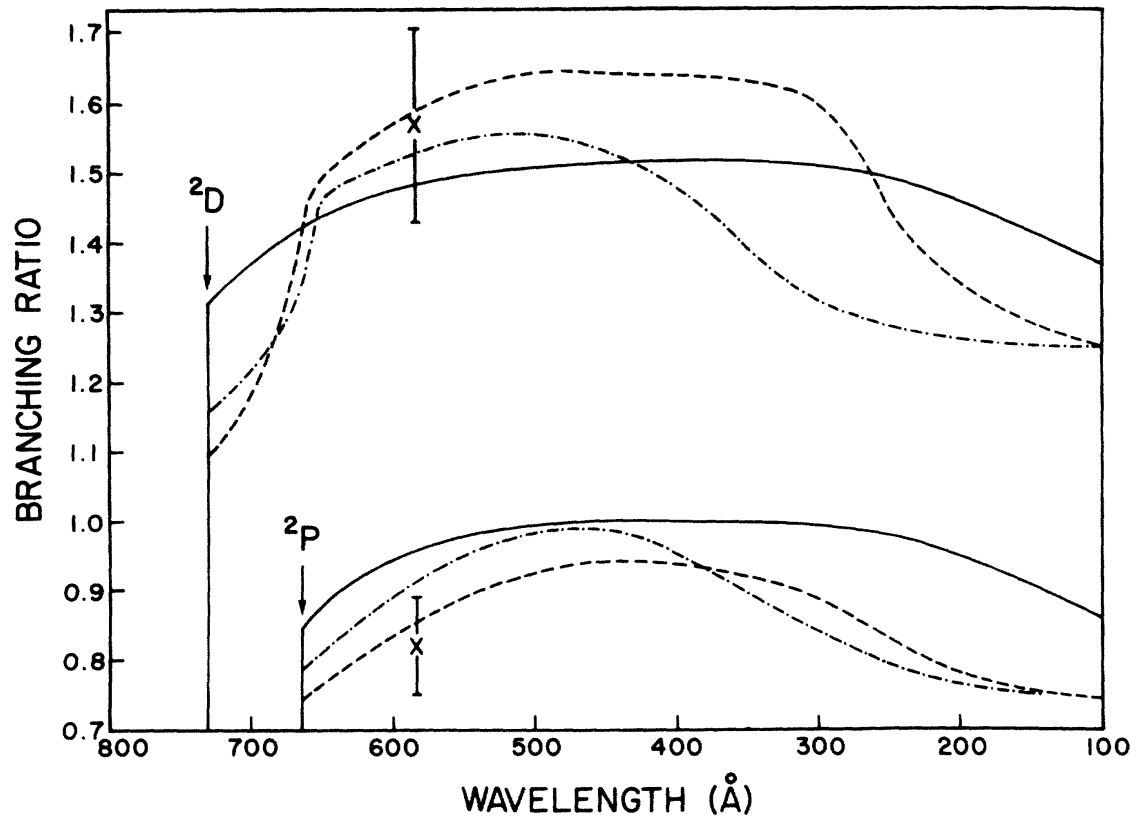

FIG. 3. Photoelectron branching ratios $\sigma\left({ }^{2} D\right) /$ $o\left({ }^{4} S\right)$ and $\sigma\left({ }^{2} P\right) / \sigma\left({ }^{4} S\right)$ for atomic oxygen. Solid line: HS. Dash-dot line: Henry (length). Dashed line: Henry (velocity). Crosses: experimental values of Samson and Petrosky. compares with the experimental value of 0.82 $\pm 0.07 .{ }^{11}$ It is clear from Fig. 3 and Table III that experimental measurements of photoelectron branching ratios at a variety of wavelengths would be of great interest to atomic theorists.

\section{CONCLUSIONS}

We have confirmed the prediction of Dill, Manson, and Starace ${ }^{1}$ that examination of photoelectron phase-shift differences is the appropriate criterion for determining how elaborate a calculation must be made for obtaining accurate angular distribution asymmetry parameters for open-shell atoms. We have found that HF photoelectron phase shifts in atomic oxygen are sufficiently close to one another to permit a simple calculation of the asymmetry parameters for $2 p$ photoionization. We expect our results to apply generally to atoms having open $2 p$ subshells.

We have also calculated total and partial photoionization cross sections for atomic oxygen using both Herman-Skillman and Hartree-Fock wave functions. At $584.3 \AA$ our calculated branching ratios are in good agreement with the experimental measurements of Samson and Petrosky. ${ }^{11}$ They compare well with other theoretical cross-section calculations except the more detailed close-coupling calculations of Henry. ${ }^{7}$ It would be of great theoretical interest therefore to employ close-coupling wave functions to calculate asymmetry parameters within the framework of Ref. 1.

\section{ACKNOWLEDGMENTS}

One of us (A.F.S.) wishes to thank Professor J. A. R. Samson for suggesting the study of atomic oxygen.
*Supported in part under National Aeronautics and Space Administration Contract No. NGR 28-004-021.

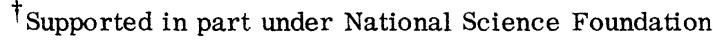
Contract No. GP-38905.

$\$$ Present address: School of Physical Sciences, New University of Ulster, Coleraine, Northern Ireland.

${ }^{1}$ D. Dill, S. T. Manson, and A. F. Starace, Bull. Am. Phys. Soc. 18, 1514 (1973); Phys. Rev. Lett. (to be published).

${ }^{2} \mathrm{~J}$. Cooper and R. N. Zare, Lectures in Theoretical Physics, edited by S. Geltman, K. T. Mahanthappa, and W. E. Britton (Gordon and Breach, New York, 1969), Vol. XI-C, pp. 317-37. The limitations of the Cooper-Zare theory, presumably well-known to the authors, arise essentially from the authors' initial as- sumption that the radial part of the photoelectron wave function is independent of photoelectron channel quantum numbers.

${ }^{3}$ F. Herman and S. Skillman, Atomic Structure Calculations (Prentice-Hall, Englewood Cliffs, N. J., 1963).

${ }^{4} \mathrm{~S}$. T. Manson, J. Electron Spectroscopy and Related Phenomena 1, 413 (1973).

${ }^{5}$ D. R. Bates and M. J. Seaton, Mon. Not. Roy. Astron. Soc. 109,698 (1949).

${ }^{6}$ A. Dalgarno, R. J. W. Henry, and A. L. Stewart, Planet. Space Sci. 12, 235 (1964).

${ }^{7}$ R. J. W. Henry, Planet. Space Sci. 15, 1747 (1967).

${ }^{8}$ G. M. Thomas and T. M. Helliwell, J. Quant. Spectrosc. Radiat. Trans. 10, 423 (1970).

${ }^{9}$ H. Kähler, J. Quant. Spectrosc. Radiat. Transfer $\underline{11}$, 
1521 (1971).

${ }^{10}$ P. S. Ganas, Phys. Rev. A 7, 928 (1973).

${ }^{11} \mathrm{~J}$. A. R. Samson and V. E. Petrosky, preceding paper, Phys. Rev. A 9, 2449 (1974).

${ }^{12}$ U. Fano and D. Dill, Phys. Rev. A $\underline{6}, 185$ (1972).

${ }^{13}$ D. Dill and U. Fano, Phys. Rev. Lett. 29, 1203 (1972).

${ }^{14}$ D. Dill, Phys. Rev. A 7, 1976 (1973).

${ }^{15} \mathrm{C}$. E. Moore, Ionization Potentials and Ionization Limits Derived from the Analysis of Optical Spectra (U.S. GPO, Washington, D.C., 1970).

${ }^{16}$ E. Clementi, IBM J. Res. Develop. 9 , 2 (1965), and supplement entitled "Tables of Atomic Functions."

${ }^{17} \mathrm{D}$. J. Kennedy and S. T. Manson, Phys. Rev. A $\underline{5}, 227$ (1972).

${ }^{18}$ S. T. Manson and J. W. Cooper, Phys. Rev. $\underline{165}, 126$ (1968).

${ }^{19}$ D. R. Bates, Mon. Not. Roy. Astron. Soc. 106, 432 (1946).

${ }^{20}$ A. F. Starace, Phys. Rev. A $\underline{3}, 1242$ (1971); $\underline{8}, 1141$ (1973).

${ }^{21}$ R. B. Cairns and J. A. R. Samson, Phys. Rev. 139, A1403 (1965). 